\title{
Volumetric registration of magnetic nanoparticles for optimization of quantitative immunochromatographic assays for detection of small molecules
}

\author{
Natalia V. Guteneva ${ }^{1,2, *}$, Sergey L. Znoyko ${ }^{1}$, Alexey V. Orlov ${ }^{1,2}$, Maxim P. Nikitin ${ }^{2}$, and Petr I. Nikitin ${ }^{1,3}$ \\ ${ }^{1}$ Prokhorov General Physics Institute, Russian Academy of Sciences, 38 Vavilov St, 119991 Moscow, Russia \\ ${ }^{2}$ Moscow Institute of Physics and Technology (State University), 9 Institutskii per., 141700 Dolgoprudny, Russia \\ ${ }^{3}$ National Research Nuclear University MEPhI (Moscow Engineering Physics Institute), 115409 Moscow, Russia
}

\begin{abstract}
Precise quantitative and highly sensitive detection of small molecules (haptens) is highly demanded in medicine, food quality control, in vitro diagnostics, criminalistics, environmental monitoring, etc. In the present work, the magnetic method of particle quantification and the optical methods of spectral correlation and spectral phase interferometry complement each other for optimization of a quantitative assay for measuring concentrations of small molecules. The assay employs magnetic nanoparticles as labels in rapid immunochromatographic format. The approach was demonstrated with fluorescein as a model molecule. The interferometric label-free biosensors were employed for selection of optimal reagents that produced high specificity and sensitivity. The method of magnetic particle quantification counted the magnetic labels over the entire volume of the immunochromatographic membrane to provide their distribution along the test strip. Such distribution was used for optimization of such parameters as concentrations of the used reagents and of antibody immobilized on the labels, amount of the labels and conjugates of haptens with protein carriers to realize the advanced quantitative immunochromatographic assay.
\end{abstract}

\section{Introduction}

Small molecules (haptens), i.e., toxins, hormones, drugs, antibiotics and vitamins exhibit biological activity even in extremely low concentrations. Development of methods for ultrasensitive quantitative detection of the small molecules is among the high-priority research tasks in many fields of science and industry [1,2].

Liquid chromatography and mass-spectrometry traditionally used for detection of small molecules $[3,4]$ offer very high sensitivity and specificity. However, long sample preparation, expensive equipment and reagents significantly limit their use.

Immunological methods are simpler and more costeffective. This group includes sensitive and affordable label-free biosensors that employ microscope cover slips as single-used sensor chips [5], fluorescence polarization [6], lateral flow (LF) tests based on optical labels [7], smart polymer-based bioanalysis [8], etc. A more complicated enzyme-linked immunosorbent assay (ELISA) is the most commonly used test considered as the "gold standard" of immunological methods [9]. The quantitative ELISA-type methods for measuring hapten concentrations are relatively long (2-24 h) and laborconsuming, require re-calibrating for each measurement using standard probes. Some of the mentioned drawbacks can be overcome using the immunochromatographic (IC) approach realized with nitrocellulose LF test strips. The approach is simple, rapid, cost-effective, does not require specially trained personnel. The existing LF methods for hapten detection employ visual or instrumental registration of various optical labels such as quantum dots, fluorescent or color markers, and gold nanoparticles, which are the most popular [7]. Although numerous studies have reported quantitative readout of gold labels on the LF strips by different electronic detectors or scanners, in the majority of countries, such assays are still approved as the threshold "yes/no" tests only.

Magnetic nanoparticles (MP) employed as the labels instead of the optical markers offer substantial advantages because they can be counted on flat surfaces by magnetic field sensors built in microfluidic chips [10] or from the entire volume of $3 \mathrm{D}$ porous structures used as the solid phase. The latter solution was successfully applied for multiplex measuring in a wide dynamic range of concentrations of protein molecules [11].

Here we propose several quantitative measuring techniques as candidates for development of an analytical platform for hapten detection with magnetic labels in the competitive format. As small molecules possess only one antigen determinant, competitive binding of antibody $(\mathrm{Ab})$ with either free hapten in the test sample or that conjugated with protein deposited on the IC membrane should be recorded. Therefore, the reproducibility of such assay strongly depends on

Corresponding author: nguteneva@gmail.com 
accessibility of the hapten antigen determinant in the conjugate, which varies according to the ways of formation. For quantitative monitoring of reproducibility of these conjugates and kinetics of binding with different $\mathrm{Ab}$, the original optical biosensors have been modified. The fluorescein was used as a model small molecule for optimization of conjugate design for further application of the results for development of magnetic ultra-sensitive and quantitative LF assays.

\section{Experimental}

\subsection{Reagents}

The following reagents were used in the experiments: mouse monoclonal antibody against fluorescein (clone FCII) were purchased from the Russian Research Center of Molecular Diagnostic and Therapy (Moscow, Russia); fluorescein isothiocyanate (FITC) was purchased from Thermo Fisher Scientific; bovine serum albumin (BSA), phosphate buffered saline (PBS) tablets were purchased from Sigma-Aldrich (Germany); 198-nm Bio-Estapor microspheres were purchased from Estapor - Merck Millipore (Germany); nitrocellulose membrane NC-140 (255 $\mu \mathrm{m}$ thick and $100 \mu \mathrm{m}$ backing) was kindly donated by Sartorius AG (Germany); absorbent sinks/wicking pads were purchased from Ahlstrom CytoSep (Finland); backing cards were obtained from Lohmann (USA); N(3 - dimethylaminopropyl) - $\mathrm{N}^{\prime}$ - ethylcarbodiimide hydrochloride (EDC) was from (Fluka, Switzerland); deionized water, MilliQ grade, was from Millipore Corp. (USA). All other chemicals were of analytical grade.

\subsection{Magnetic particle quantification}

Magnetic nanolabels were counted along the LF strips by original readers based on the magnetic particle quantification (MPQ) method [11]. The MPQ employs nonlinear magnetization of MP by a magnetic field at two frequencies and records the response at combinatorial frequencies. The method is insensitive to linear dia- and paramagnetic materials such as glass, water, etc. The MPQ readers modified for these studies feature improved spatial resolution for various biophysical applications including precise quantification of MP along the LF strips. They have the limit of detection of $0.4 \mathrm{ng}$ or $60 \mathrm{zmol}$ of MP within the volume up to $0.2 \mathrm{~mL}$ and the 7 -order linear dynamic range.

\subsection{Immunochromatographic assay procedure}

Standard IC test strips were used, each being composed of overlapping sample pad, nitrocellulose and absorbent membranes assembled on an adhesive plastic backing sheet (Fig. 1). The Sartorious UniSart Cn140 membrane of $255 \mu \mathrm{m}$ thick characterized by the manufacturer was composed of cellulose nitrate polymers and exhibited capillary speed of $140 \mathrm{~s} / 40 \mathrm{~mm}$.

The conjugate of BSA with FITC at concentration of $2.5 \mathrm{mg} / \mathrm{ml}$ and goat $\mathrm{Ab}$ against mouse $\mathrm{IgG}$ (GAM Ab) at concentration of $1 \mathrm{mg} / \mathrm{ml}$ were used to form on the nitrocellulose membrane the test (TL) and control (CL) lines, respectively. Then conjugates of MP with different amounts of antibody were prepared according to the procedure described in [12]. The superparamagnetic 198-nm Bio-Estapor carboxyl-modified spheres were used as the detectable labels. These magnetic nanoparticles conjugated with antibody migrate well through the membrane delivering high and specific signal. The conjugates were added to the samples containing a known amount of fluorescein used in this study as a model and incubated for $5 \mathrm{~min}$. The main parameters of the assay such as reaction time, amount of reagents and sample volume were optimized using the MPQ readers toward higher sensitivity.

The IC test strips were placed vertically into the prepared solutions for $20 \mathrm{~min}$. Then the test strips were passed through the measuring coil of the MPQ reader to measure the signals. All the experiments were implemented at least thrice and statistically processes. In graphs, each value represents an average, and error bars show standard deviations.

\subsection{Label-free monitoring of molecular bindings}

For development of the sensitive and rapid assays, we carried out selection of optimal antibodies and conjugates of fluorescein with BSA by their binding kinetic characterization using modified label-free biosensors based on spectral-phase interferometry (SPI) [13] and spectral-correlation interferometry (SCI) [14] that provide real-time monitoring of thickness of a layer of biomolecular complexes bound on the sensor chip surface due to biochemical reactions. The techniques use microscopic cover glass slips as two-beam reflective interferometers and affordable sensor chips simultaneously. These biosensors showed high efficiency for optimization of $\mathrm{Ab}$ immobilization interfaces and development of protocols for sandwich magnetic immunoassays [14].

\section{Results and discussion}

The single-channel SPI and 3-channel SCI biosensors were used for real-time monitoring of immunochemical reactions of the reagents, as well as to control reproducibility of MP functionalization, of accessibility of antigen determinants in synthesized conjugates, etc.

The carboxylated glass cover slip was activated with EDC solution, and then FITC/BSA was covalently immobilized to it. To block the unreacted carboxyl groups on the surface, $1 \%$ glycine solution was pumped along the glass cover slip installed in the biosensor flow system followed by pumping of $\mathrm{Ab}$ solution. The examples of recorded sensograms, used to control reproducibility of antigen determinants on the conjugates and specific binding, are shown in Fig. 2. The sensograms were obtained using the label-free biosensors based on the interferometric techniques $[13,14]$ briefly described in the "Experimental" section. 


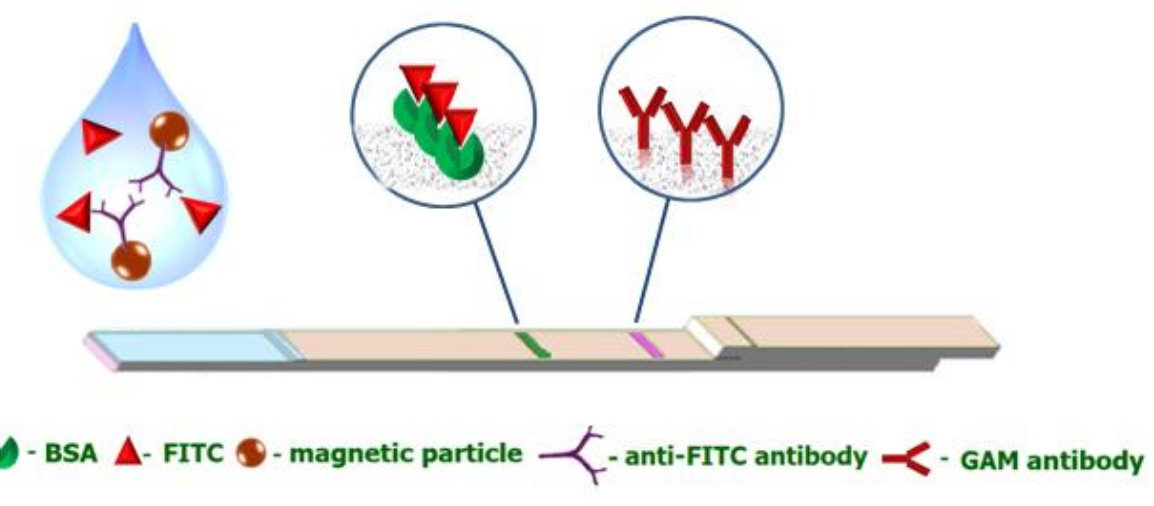

Fig. 1. Scheme of the competitive immunochromatographic assay procedure. Goat anti mouse antibody (GAM) is deposited onto the control line, the BSA/FITC conjugate deposited onto the test line competes with free antigen in sample for the limited amount of anti-FITC antibody binding sites.

The sensograms show the temporal dependence of $\Delta \mathrm{d}$, which is a change in thickness of the biomolecular layer on the sensor chip surface averaged over the measuring area. Two test samples were used: i) without haptens so that free $\mathrm{Ab}$ binds to the conjugate and ii) with haptens that binds to all $\mathrm{Ab}$ in solution preventing binding with the conjugate. The same experiment was repeated for different concentrations of antibody solutions and immobilized FITC/BSA conjugates.

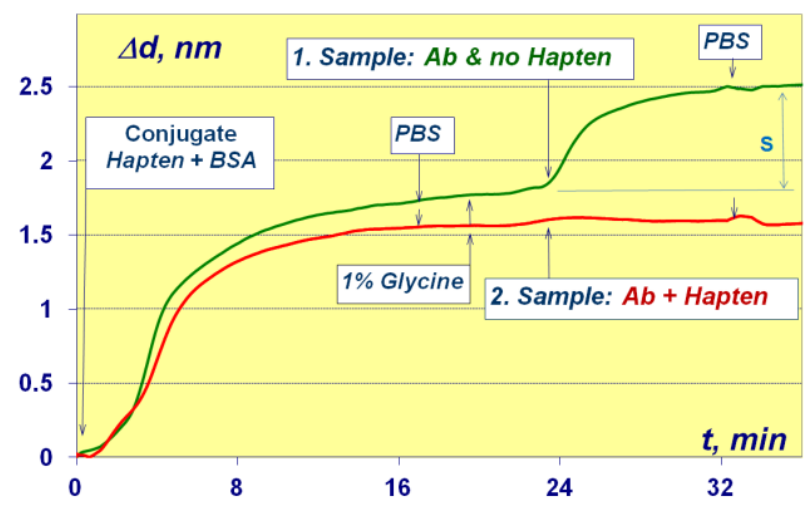

Fig. 2. Sensograms of competitive label-free assay in the presence and absence of haptens.

The observed difference $\mathrm{S}$ in the signal is traditionally used as assay result. It depends on availability and accessibility of antigen determinants on the conjugate. To get the results faster, the $\mathrm{Ab}$ were selected, which demonstrated the steepest slope of the sensograms.

New efficient quantitative methods of assay optimization have been proposed based on the developed technique of magnetic detection. One of them is based on local counting of MP over the entire depth of the strip and mapping of MP distribution (Fig. 3) along all the constituent components of lateral flow test strips (not merely on the membrane surface of TL and CL as it is typically done with optical labels). The MP distribution was used for optimization of assay parameters such as $\mathrm{Ab}$ immobilized on MP, $\mathrm{pH}$ of buffers, sample volume, amounts of MP and conjugates deposited onto the test lines to achieve highest sensitivity. According to the experiments, the optimal values were: $\mathrm{pH}-7.5$, MP amount - $3 \mu \mathrm{g}$, and sample volume - $75 \mu \mathrm{l}$.

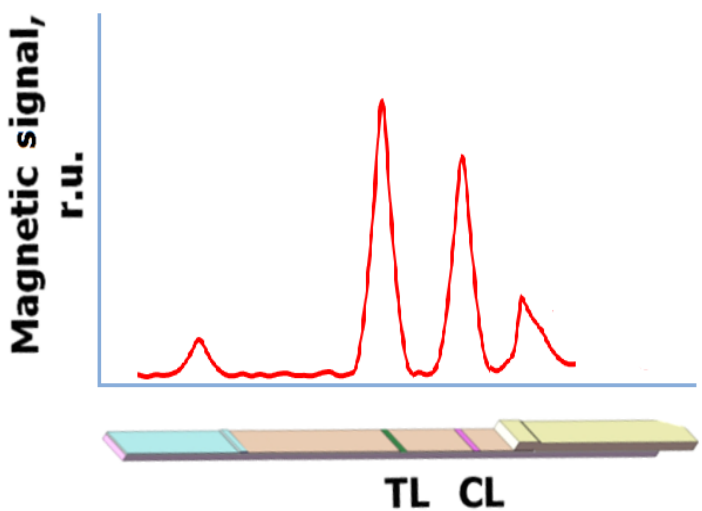

Fig. 3. Distribution of magnetic nanolabels along the test strip measured by the MPQ reader. The signal is proportional to the local amount of magnetic nanoparticles.

The optimization procedure is illustrated here for amount of Ab immobilized on MP, which is among the most important parameters that may strongly influence the assay sensitivity. As small molecules are detected here in the competitive format, if excessive amount of $\mathrm{Ab}$ is present in the test sample, then the antigen concentration does not practically affect the magnetic signal because MP bind to the test line anyway. On the contrary, insufficient amount of $\mathrm{Ab}$ conjugated with MP yields a weak signal on the test line due to low-effective binding of MP, and this significantly reduces the assay sensitivity. The examples of dependences of magnetic signal upon amount of $\mathrm{Ab}$ conjugated with MP obtained with the proposed method are shown in Fig. 4.

One may see from the Fig. 4 that the best ratio of the signal in presence and absence of fluorescein corresponds to $3 \mu \mathrm{g}$ of $\mathrm{Ab}$ immobilized on MP. As expected, in case of insufficient amount of $\mathrm{Ab}$ on MP $(0.3 \mu \mathrm{g})$, the magnetic signal registered on the TL is too weak for sensitive detection of antigen. Otherwise, when large amount of $\mathrm{Ab}$ on MP $(30 \mu \mathrm{g})$ is used, the magnetic signals in presence and absence of fluorescein are almost equal, and that hinders detection of the small molecules. 

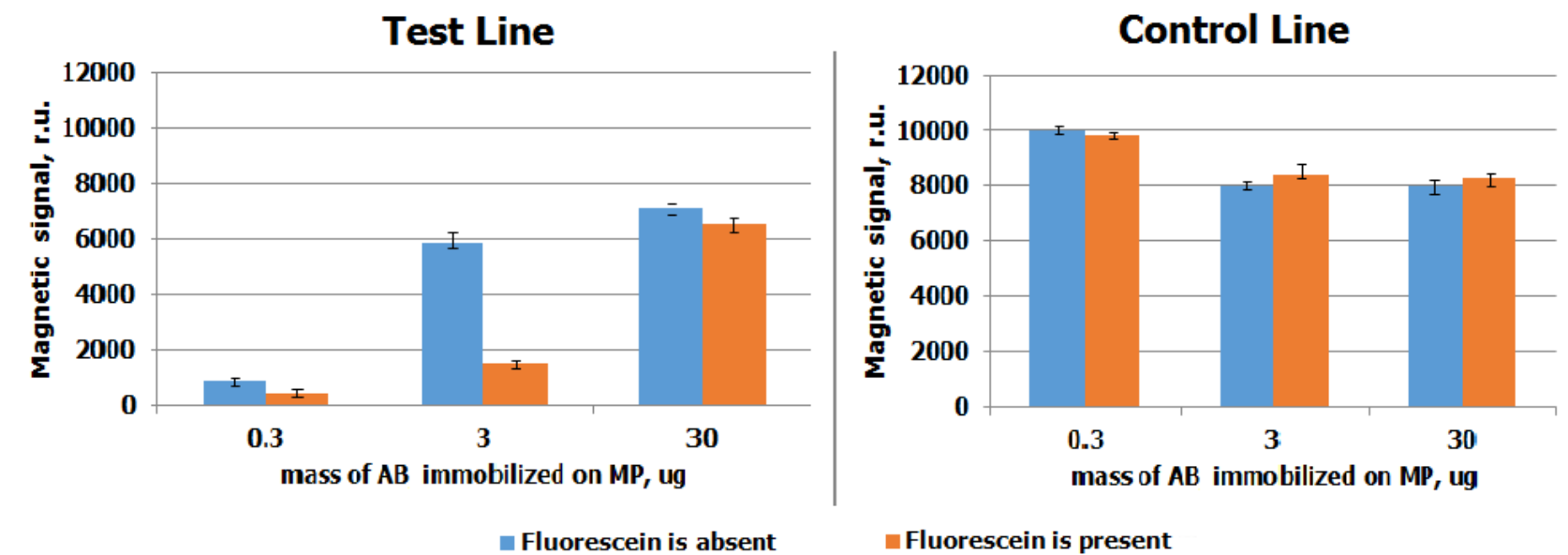

Fig. 4. Dependences of magnetic signal from MPQ reader on test and control lines upon amount of antibody conjugated with magnetic nanoparticles in the presence and absence of antigen.

Thus, the proposed method of MP distribution mapping provides a tool for rapid, simple and costefficient optimization of all stages of the IC assay without high consumption of reagents. The method has been also used for quantitative monitoring of total MP mass by determination of square under the curve of their distribution along the test strip. According to the experiments, this parameter did not depend on antigen concentration and remained constant for each batch of the test strips. The method for optimization of quantitative IC assays for detection of small molecules can be used for rapid and cost-effective development of highly sensitive express test systems for food quality control, in vitro diagnostics, criminalistics, environmental monitoring, etc.

\section{Conclusion}

The proposed method of recording quantitative distribution of magnetic nanoparticles along all constituent components of immunochromatographic lateral flow strips can be used for selection of proper concentrations of the used reagents such as amounts of $\mathrm{Ab}$ immobilized on MP, amounts of MP and conjugates deposited onto the test lines, etc.

The advanced label-free optical biosensors based on the SPI and SCI have allowed rapid selection of optimal reagents that feature high affinity and specificity. They also have shown high efficiency to control MP functionalization and synthesis of conjugates, interaction of conjugate with specific $\mathrm{Ab}$, etc. These magnetic and optical methods are also promising for transformation of the previously reported magnetic LF sandwich assays for sensitive detection of small molecules in a wide dynamic range.

The experiments have demonstrated that the synergetic combination of original highly sensitive magnetic instruments having extraordinarily wide 7order dynamic range with interferometric methods yields powerful tools for development of advanced analytical systems based on magnetic nanolabels.
Different aspects and parts of this multidisciplinary research were partially supported by the Russian Foundation for Basic Research (grants No. 16-33-60228, 15-04-09372, 15-32-70022, 15-04-99499, 15-02-07791, 17-54-560024, 17-02-01415) and the Competitiveness Programs of NRNU "MEPhI" (P.I.N.).

\section{References}

1. X. Wang and S. Wang, Sensors, 8, 6045 (2008)

2. D.R. Shankaran, K.V. Gobi, N. Miura, Sensors and Actuators B: Chemical, 121, 158 (2007)

3. E. González-Peñas, C. Leache, M. Viscarret, A. Pérez De Obanos, C. Araguás, A. López de Cerain, J. Chromatogr. A, 1025, 163 (2004)

4. A.M. Evans, C.D. Dehaven, T. Barrett, M. Mitchell, E. Milgram, Anal. Chem. 81, 6656 (2009)

5. A.V. Orlov, A.G. Burenin, N.G. Massarskaya, A.V. Betin, M.P. Nikitin, P.I. Nikitin, Sensors and Actuators B: Chemical, 246, 1080 (2017)

6. S.C. Pei, Y.Y. Zhang, S.A. Eremin, W.J. Lee, Food Control 20, 1080 (2009)

7. D. Kong, L. Liu, S. Song, S. Suryoprabowo, A. Li, .H. Kuang, L. Wang, C. Xu, Nanoscale, 8, 5245 (2016)

8. A.E. Ivanov, V.P. Zubov, Russ. Chem. Rev. 85, 565 (2016)

9. D. Wild, The Immunoassay Handbook, 4th edn. (Elsevier Science, 2013)

10. I. Giouroudi, G. Kokkinis, Nanomaterials, 7, 171 (2017)

11. A.V. Orlov, S.L. Znoyko, V.R. Cherkasov, M.P. Nikitin, P.I. Nikitin, Anal. Chem. 88, 10419 (2016)

12. G.T. Hermanson Bioconjugate techniques, 3rd edn. (Academic, New York, 2013)

13. P.I. Nikitin, B.G. Gorshkov, M.V Valeiko, S.I. Rogov, Quantum Electronics, 30, 1099 (2000)

14. A.V. Orlov M.P. Nikitin, V.A. Bragina, S.L. Znoyko, M.N. Zaikina, T.I. Ksenevich, B.G. Gorshkov, P.I. Nikitin, J. Magn. Magn. Mater. 380, 231 (2015) 